\title{
Restrictive dermopathy: a report of three cases
}

\author{
Q Mok, R Curley, J L Tolmie, R A Marsden, M A Patton, E G Davies
}

\begin{abstract}
We report three infants with a rare syndrome of restrictive dermopathy, in which rigidity of the skin at birth is associated with characteristic facial anomalies, generalised arthrogryposis, bony abnormalities, and lung hypoplasia. The skin has a distinctive pathology with compaction of the dermal collagen and fibrosis of the subcutaneous tissue. The inheritance is likely to be autosomal recessive and the condition appears to be fatal in the early neonatal period.
\end{abstract}

Several cases of the fetal akinesia/hypokinesia deformation syndrome have been described, where facial anomalies, arthrogryposis, and lung hypoplasia are associated. Examples include Pena-Shokier, NeuLaxova, cerebro-oculo-facio-skeletal, and lethal

Departments of Child Health, Dermatology, and Medical Genetics, St George's Hospital Medical School, London. Q Mok, R Curley, R A Marsden, M A Patton, E G Davies

Duncan Guthrie Institute of Medical Genetics, Glasgow. J L Tolmie

Correspondence to Dr Mok, Queen Mary's Hospital for Children, Carshalton, Surrey SM5 4NR.

Received for publication 12 October 1989.

Revised version accepted for publication 18 December 1989. multiple pterygium syndromes. Restrictive dermopathy is a distinctive and recently described disorder in which the primary defect appears to be rigidity of the skin, causing secondary generalised flexion contractures and restriction of respiratory movements, thus leading to early death.

\section{Case reports \\ CASE 1}

Case 1 was the first child of healthy, non-consanguineous, Caucasian parents. The mother, aged 24 years, did not smoke or drink and was not exposed to any known teratogens. The family history is otherwise unremarkable. The pregnancy proceeded normally until spontaneous rupture of membranes at $28+$ weeks' gestation, when an ultrasound scan showed a normal amount of liquor and no fetal breathing movements. Despite treatment with ritodrine and dexamethasone labour progressed and five days later a male infant was delivered with difficulty by forceps. He had an Apgar score of 2 at one minute and was intubated at two minutes with difficulty because of a small, tight mouth and restriction in neck extension. The placenta weighed $530 \mathrm{~g}$ and had three vessels.

The infant weighed $1080 \mathrm{~g}$ (above the 10th centile) with an occipitofrontal circumference (OFC) of $27 \mathrm{~cm}$ (below the 50th centile). He had a dorsal kyphosis, rockerbottom feet, and contractures of all the joints (fig 1) including the fingers and mandible. The facial

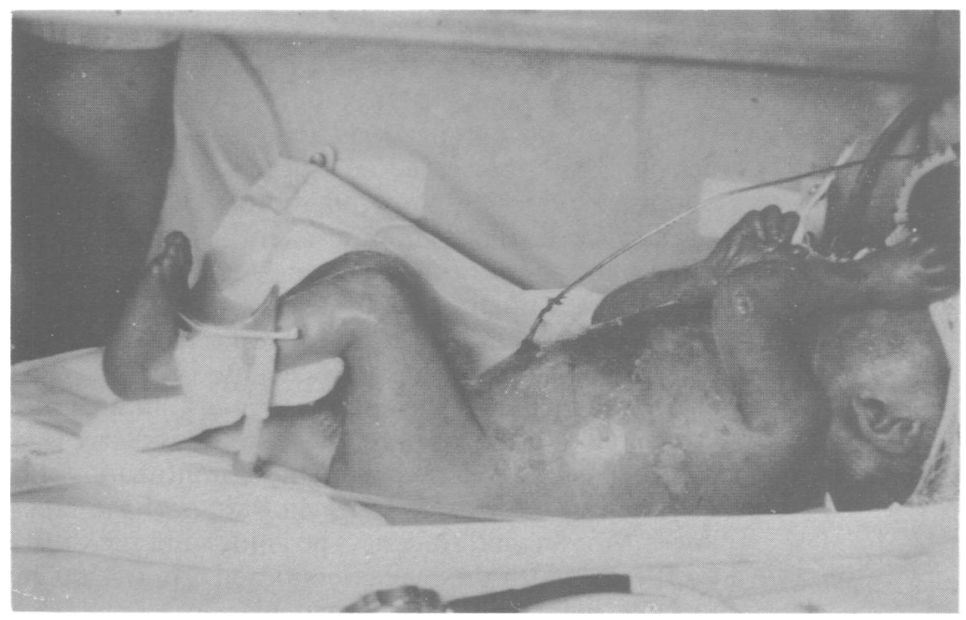

Figure 1 Case 1 showing severe contractures of all the joints. 
appearance was distinctive with an antimongoloid slant and hypertelorism, small pinched nose, microstomia with a small, open, $\mathrm{O}$ shaped mouth, and micrognathia (fig 2). The anterior fontanelle was large with wide sutures. At birth he was noted to have smooth, shiny skin which felt rigid and tethered. The cutaneous vasculature was easily visible, suggesting thinning of the dermis. Within hours of birth, fragile blisters had developed over the anterior abdominal wall and extensor aspect of the elbows, and these later ruptured to leave superficial erosions (fig 3 ). The hair and nails were normal. Initially adequate gas exchange was easily obtained on low pressure ventilation but his lungs became progressively more difficult to ventilate. The skin continued to break down readily and became more rigid.

Karyotyping showed normal 46,XY chromosomes. $X$ rays showed deficient ossification of the distal clavicles with segmentation, and the long bones, especially the humeri, showed 'overmodelling' (fig 4). A skin biopsy from the edge of a blister showed parakeratosis of the epidermis and a subepidermal split. The dermis appeared compacted with loss of the normal space between collagen bundles, and there were striking downward projections of fibrous tissue extending from the dermis into the subcutaneous fat.

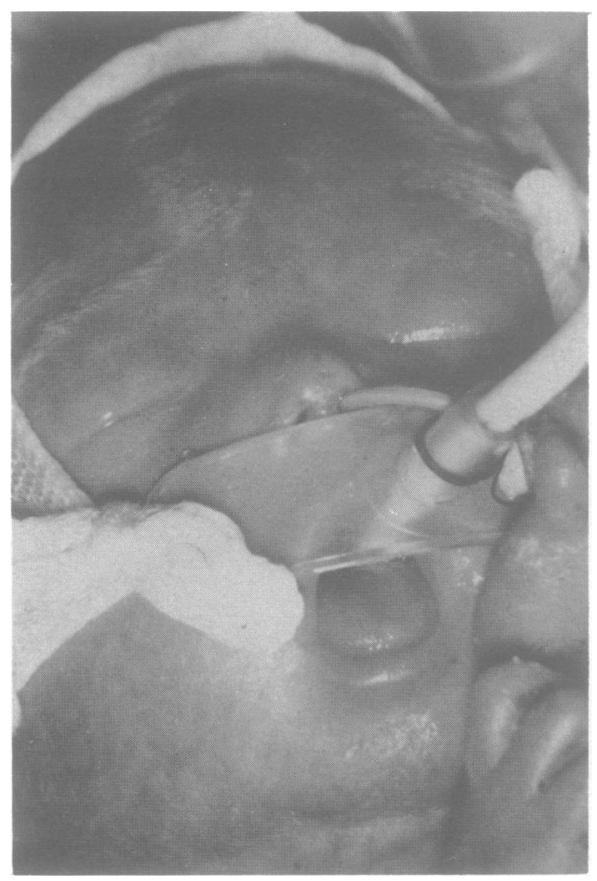

Figure 2 The distinctive facial appearance of a case of restrictive dermopathy. Note the antimongoloid slant and hypertelorism, small pinched nose, microstomia with a small, open, $O$ shaped mouth, and micrognathia.

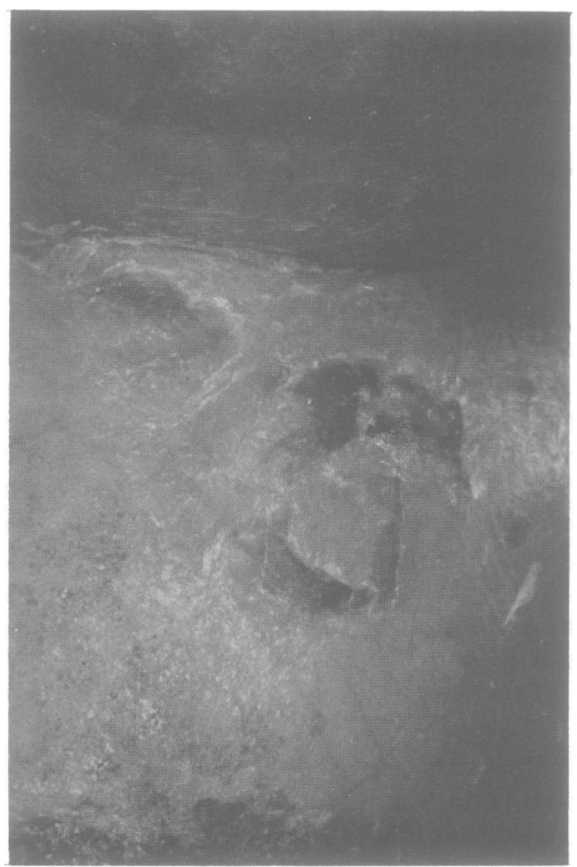

Figure 3 Close up of the skin showing the superficial erosions.

The adipose tissue itself was increased in amount and contained proliferating fibroblasts (fig 5).

Supportive treatment was withdrawn in view of the deteriorating condition and poor prognosis, and the infant died aged 1 week.

\section{CASE 2}

Case 2 was the second child of consanguineous Pakistani parents. His mother, aged 21 years, took triiodothyronine for hypothyroidism and remained euthyroid throughout pregnancy. The pregnancy was normal until 32 weeks' gestation when spontaneous rupture of membranes was followed by a vaginal delivery. The infant had an Apgar score of 2 at one minute with no response to intubation and resuscitation. He died at 30 minutes. The placenta weighed $450 \mathrm{~g}$ and was uniformly pale, thick, and oedematous.

The infant weighed $1300 \mathrm{~g}$ (below the 10th centile) with an OFC of $27 \mathrm{~cm}$ (below the $3 \mathrm{rd}$ centile). He had an antimongoloid slant, pinched nose, microstomia, and micrognathia. The skin was taut and shiny, and a horizontal split in the skin over the thyroid cartilage had been noted even before attempts at intubation (fig 6). There were fixed flexion deformities of the hips, knees, elbows, and fingers. The ankles and the third, fourth, and fifth toes were dorsiflexed. He was noted to have long nails and normal hair. 


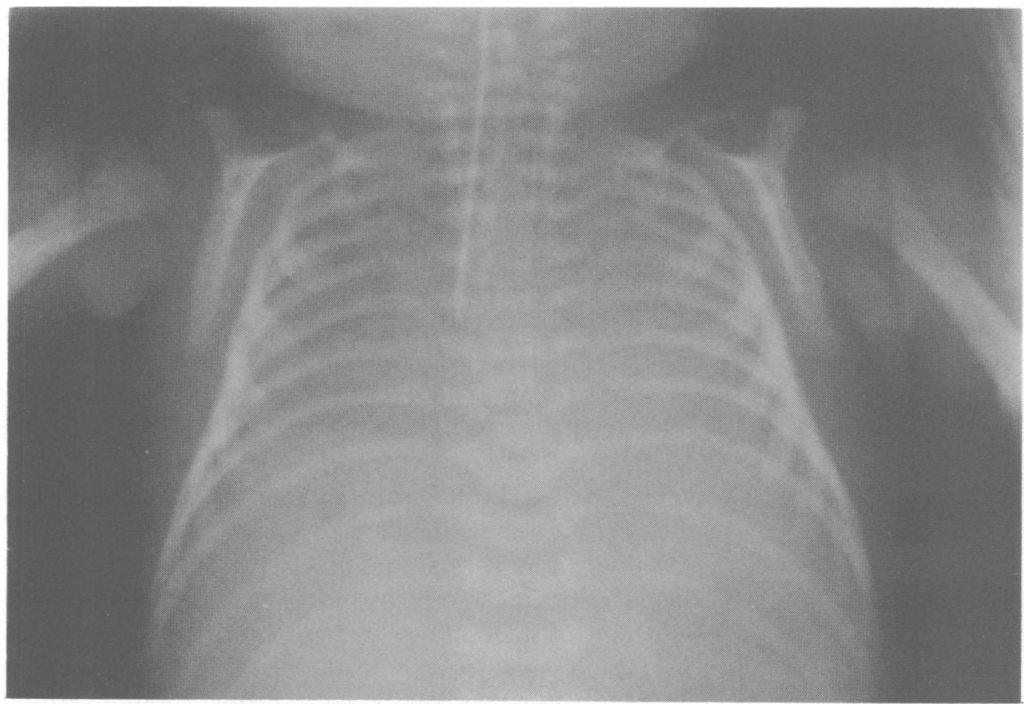

Figure 4 The $x$ ray illustrates the segmentation of the clavicles and overmodelling of the humeri, also the typical appearance of hypoplastic lungs.

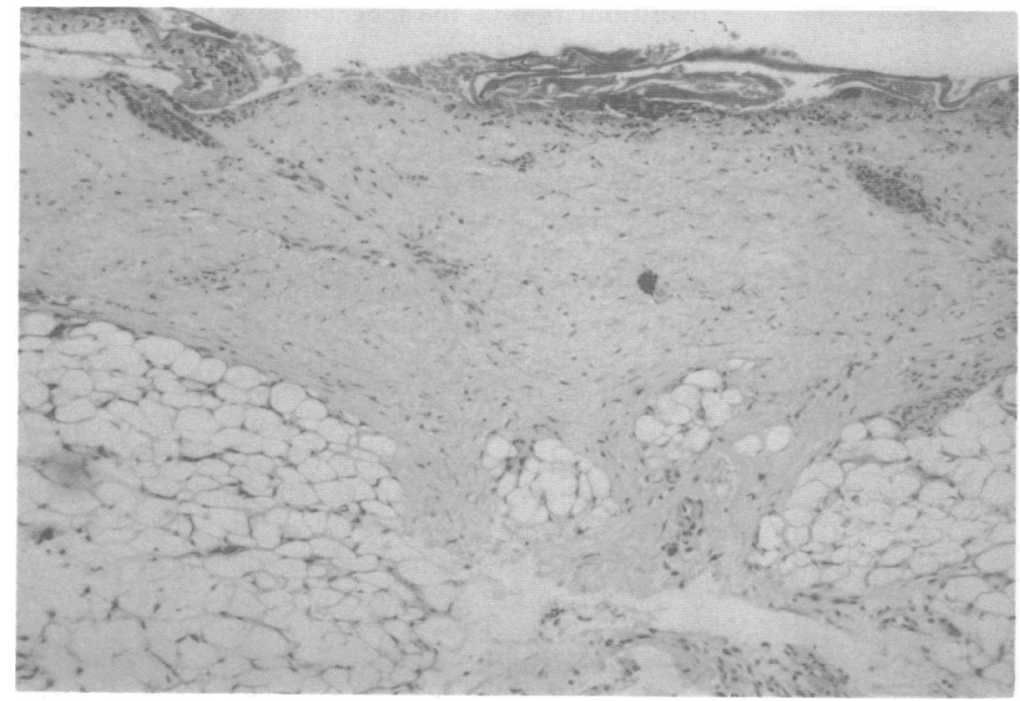

Figure 5 The skin biopsy shows a compacted dermis with downward projections of fibrous tissue into the thickened subcutaneous layer of adipose tissue.

Karyotyping (Giemsa banded) showed 46,XY chromosomes. $X$ rays showed decreased ossification of the distal ends of the clavicles, and 'overmodelling' of the long bones. A skin biopsy was not performed.

\section{CASE 3}

Case 3 was the sib of case 2 . She was born at 32 weeks after an uneventful pregnancy. Apgar scores were 5 at one minute and 7 at five minutes. She weighed $1500 \mathrm{~g}$ (10th centile) with an OFC of $29 \mathrm{~cm}$ (10th centile).
She had exactly similar dysmorphic features to the previous infant (fig 7). The skin was of similar appearance and texture, the nails were long, and there were flexion contractures of the joints.

She had a normal $46, \mathrm{XX}$ karyotype. $X$ ray findings were similar to the previous infant. A skin biopsy showed acanthosis and hyperkeratosis of the epidermis with reduction in papillary dermis, widespread telangiectasia, and poorly formed hair and sweat gland elements.

The infant required tube feeds and over the 


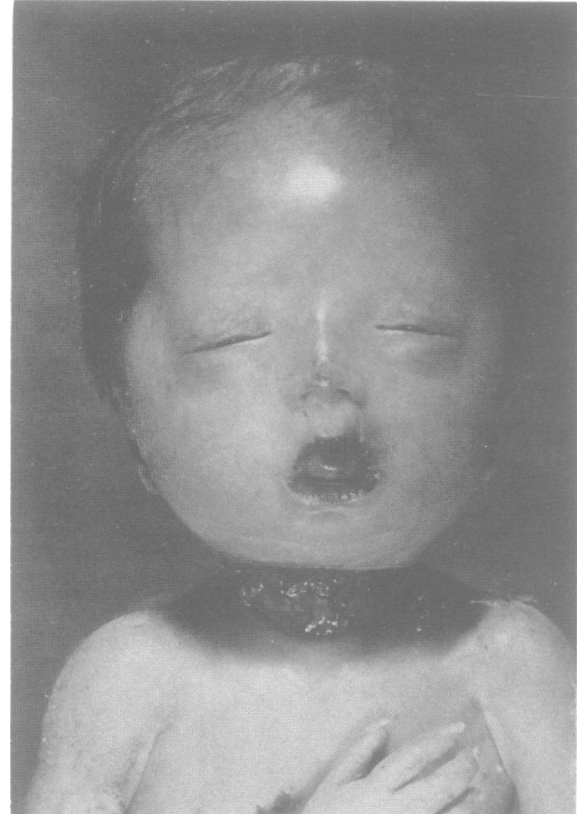

Figure 6 A close up of case 2 showing the typical facial features and the split in the skin over the thyroid cartilage that was present from birth.

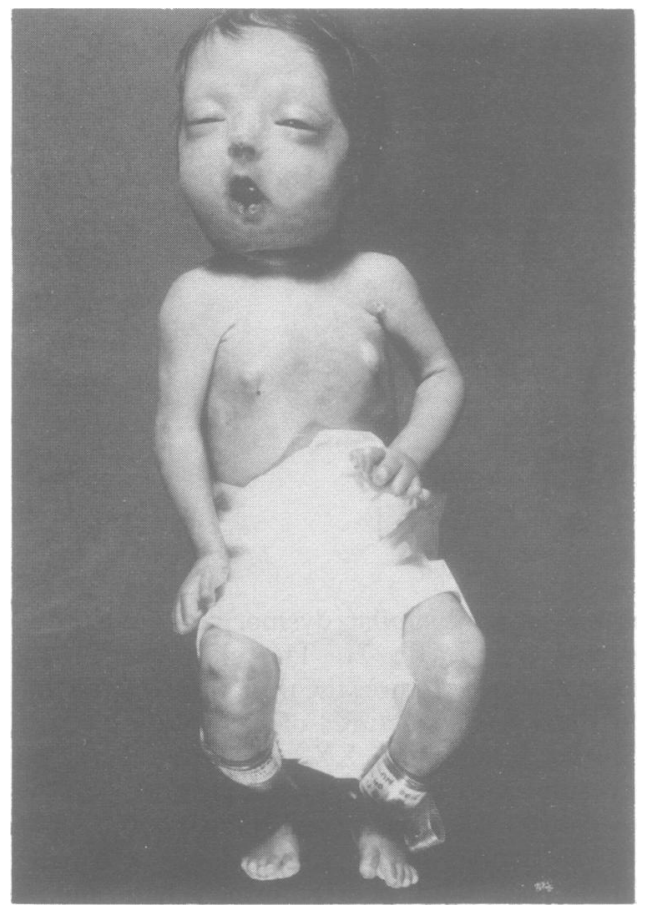

Figure 7 The postmortem photograph of case 3 showing all the features of the condition previously described. following six weeks the skin became progressively less elastic. Three primary teeth erupted prematurely. Cyanotic attacks became more frequent and the infant died aged 7 weeks.

The parents had a previous healthy male child and a subsequent healthy female child. They also had two subsequent intrauterine deaths at 16 and 26 weeks, respectively.

Necropsy was refused in all three cases.

\section{Discussion}

The term 'restrictive dermopathy' was first used by Witt $e t a l^{1}$ to describe two sibs from consecutive pregnancies, both born prematurely at 32 weeks. They shared the clinical, radiological, and histological features of our three infants with the characteristic facial anomalies (microstomia, micrognathia, hypertelorism, pinched nose, low set ears, wide sutures and fontanelles), arthrogryposis, pulmonary hypoplasia, and intrauterine growth retardation. Polyhydramnios was noted in both pregnancies and the infants had short umbilical cords. $X$ ray examination showed overtubulation of the long bones with segmentation defects of the clavicles.

In a more extensive study of the two sibs and a further unrelated infant, Holbrook $e t a l^{2}$ defined histological, ultrastructural, and biochemical abnormalities of the skin. They, like our infants, had shiny, rigid skin with distinctive histology showing parakeratotic hyperkeratosis, epidermal acanthosis, a dermis composed of dense parallel connective tissue, and a thickened and fibrotic layer of subcutaneous fat. Hair follicle development appeared to have been arrested at a stage corresponding to the follicle development in the first or early second trimester fetus. Epidermal extracts, when examined by gel electrophoresis and stained with antikeratin monoclonal antibodies, showed a pattern suggestive of a hyperproliferative state with an increased expression of 48 and $56 \mathrm{kD}$ keratins. Immunohistochemistry of the epidermis showed a distinctive staining pattern with the monoclonal antibody AEl, which normally stains basal cells, but in the two affected infants stained only suprabasal cells.

A number of other infants with very similar features have been reported previously. All showed the typical facial anomalies, joint contractures, and skin fragility. Although Toriello et $a l^{3}$ described two sibs as having aplasia cutis congenita, their description and photographs closely resemble our cases. Necropsy showed evidence of prematurity with patent arterial ducts, immature brain gyral pattern, and fetal lobulations of the kidneys. Toriello ${ }^{4}$ subsequently reported a third sib born to the same family with all the typical anomalies. Studies of the skin by Holbrook et $a l^{2}$ clearly showed that the infant has the same condition as the two sibs described by Witt $e t a l^{1}$ and 
termed restrictive dermopathy. The four infants reported by Lowry $e t a l^{5}$ showed no internal abnormalities apart from pulmonary hypoplasia. One of their cases also showed premature eruption of teeth and had two central incisors. Toriello ${ }^{4}$ noted remarkable consistency in the facial phenotypes in the cases described, while the skin anomaly exhibits more variable expression. The striking similarity of the cases caused Gillerot and Koulischer ${ }^{6}$ to review their patient's diagnosis and recall the family five years later to correct recurrence risk figures and propose prenatal diagnosis.

Schnur et $a l^{7}$ identified large amounts of proline and hydroxyproline containing small peptides in the skin of an affected infant, and suggested a primary defect of collagen or its metabolism. Lowry $e t a l^{5}$ and Witt $e t a l^{1}$ postulated that all the abnormalities were related to a basic disorder of skin differentiation. Rigidity of the skin caused severe restriction of movement in utero, and consequent contractures. The facial changes may be the result of the intrinsically abnormal mechanical effects of the skin, the pulmonary hypoplasia the result of limitation of chest excursion and breathing movements, and the polyhydramnios the result of abnormal swallowing. Toriello ${ }^{4}$ suggested that it is a more complex dysplasia affecting several tissues and, owing to its effect on fetal movement, numerous deformations are caused.

Restrictive dermopathy is a universally fatal condition in the neonatal period. Efforts should therefore be directed towards confirming the diagnosis rapidly after birth and minimising trauma to the infant and family.

All the reported cases suggest autosomal recessive inheritance, which is supported by a history of parental consanguinity in many of the families. In view of the high risk of recurrence in subsequent pregnancies, there is a clear need for prenatal diagnosis in potentially affected infants. Regular detailed ultrasound scanning after 16 weeks could identify decreased fetal movement and joint contractures, but may be too non-specific in determining affected cases for therapeutic abortions.

All the epithelial structural proteins are expressed by 14 to 16 weeks' gestation. Examination of fetal skin obtained after this time for morphological abnormalities and for the increased expression of the 48 and 56 $\mathrm{kD}$ keratins might be used for prenatal diagnosis. The prospects for prenatal diagnosis will improve if the specific gene defect in this condition can be determined.

We thank Dr T L Turner for permission to report cases 2 and 3 .

1 Witt DR, Hayden MR, Holbrook KA, Dale BA, Baldwin VJ, Taylor GP. Restrictive dermopathy: a newly recognized autosomal recessive skin dysplasia. Am $\mathcal{F}$ Med Genet 1986;24: $631-48$.

2 Holbrook KA, Dale BA, Witt DR, Hayden MR, Toriello HV. Arrested epidermal morphogenesis in three newborn infants with a fatal genetic disorder (restrictive dermopathy). $\mathcal{F}$ Invest Dermatol 1987;88:330-9.

3 Toriello HV, Higgins JV, Waterman DF. Autosomal recessive aplasia cutis congenita-report of 2 affected sibs. Am $\mathcal{F}$ Med Genet 1983;15:153-6.

4 Toriello HV. Invited editorial comment: restrictive dermopathy and report of another case. Am F Med Genet 1986;24:625-9.

5 Lowry RB, Machin GA, Morgan K, Mayock D, Marx L. Congenital contractures, edema, hyperkeratosis, and intrauterine growth retardation. Am F Med Genet 1985;22:531-43.

6 Gillerot Y, Koulischer L. Letter to the editor. Restrictive dermopathy. Am $\mathcal{F}$ Med Genet 1987;27:239-40.

7 Schnur RE, Ashmead J, Kelley RI. A lethal ichthyosis variant with arthrogryposis. Am $\mathcal{F}$ Hum Genet 1985;37:76A. 